\title{
In situ Ion Irradiation and Heating Experiments in the Transmission Electron Microscope: Simulations of Dust Processing in Circumstellar Environments
}

\author{
Pierre Haenecour $^{1 *}$, Michelle S. Thompson ${ }^{2}$, Thomas J. Zega ${ }^{1,3}$, Jane Y. Howe ${ }^{4}$ and Wei-Ying Chen ${ }^{5}$ \\ 1. Lunar and Planetary Laboratory, The University of Arizona, Tucson, AZ, USA. \\ 2. Dept. of Earth, Atmospheric, and Planetary Sciences, Purdue University, West Lafayette, IN, USA. \\ 3. Dept. of Materials Science and Engineering, The University of Arizona, Tucson, AZ, USA. \\ 4. Dept. of Materials Science and Engineering, and Department of Chemical Engineering and Applied \\ Chemistry, University of Toronto, Ontario, Canada. \\ 5. Applied Materials Division, Argonne National Laboratory, Lemont, IL, USA. \\ * Corresponding author: pierre@lpl.arizona.edu
}

Laboratory analyses of circumstellar (presolar) silicates and oxides provide unique insights into the origin and processing history of dust in their parent stars, e.g. [1]. In particular, coordinated NanoSIMS and transmission electron microscopy (TEM) analysis of presolar grains have revealed a wide range of chemical compositions and microstructures that are not predicted by model fits to astronomical spectra or theoretical condensation calculations, e.g. [1]. These observations provide unique constraints on dust condensation processes and thermodynamics in stellar outflows that are simply not accessible from astronomical observations, such as proportions of amorphous and crystalline grains. While the large majority of oxide grains $(84 \%)$ are crystalline, more than half of all circumstellar silicate grains analyzed with TEM [2-6 and references therein] are amorphous or are composite assemblages. This observation could either reflect the original thermodynamic conditions in which these dust grains condensed (e.g., formation of amorphous grains), or subsequent processing in circumstellar environments or the interstellar medium (ISM) due to irradiation and/or thermal alteration. Indeed, previous studies have suggested that crystalline grains might have been amorphized in circumstellar environments and/or in the ISM [7, 8]. To investigate the effects of irradiation and thermal alteration in circumstellar environments, we carried out in-situ irradiation and heating experiments on olivine $\left((\mathrm{Mg}, \mathrm{Fe}) \mathrm{SiO}_{4}\right)$ and hibonite $\left(\mathrm{CaAl}_{12} \mathrm{O}_{19}\right)$ analogs in the TEM.

San Carlos (SC) olivine and synthetic hibonite grains were crushed into a fine-grained powder in an agate mortar. After suspension in methanol, the particles were deposited onto both lacey carbon film supported on $\mathrm{Cu}$ TEM grid and onto Norcada heating chips. The samples were irradiated using Xe and He ions at Argonne National Laboratory in the intermediate voltage electron microscope tandem (IVEM) facility. We used a NEC ion implanter (Xe) and He implanter for in situ irradiation in a 300 $\mathrm{keV}$ Hitachi H9000 TEM. Both olivine and hibonite particles on the $\mathrm{Cu}$ grid were irradiated with 500 $\mathrm{keV}$ Xe ions up to 0.5 and 1 displacements per atom (dpa), respectively. The heating chip with hibonite grains was exposed to the same conditions up to a dose of $1 \mathrm{dpa}$, but the chip with SC olivines was irradiated with $20 \mathrm{keV} \mathrm{He}$ ions at room temperature to a fluence of $2 \times 10^{16} \mathrm{ions} / \mathrm{cm}^{2}$. We imaged the samples at several intervening doses during the irradiation process. Following the irradiation, the chemical composition and microstructure of all the samples were then analyzed in a Hitachi SU9000 30 $\mathrm{keV}$ STEM/SEM at the University of Arizona. The SU9000 is equipped with secondary electron, brightfield and dark-field detectors, Oxford X-Max 100TLE energy-dispersive X-ray spectrometer (EDS), and a Hitachi electron energy-loss spectrometer (EELS). The SC olivine and synthetic hibonite grains on the heating chips were then flash-heated to $900^{\circ} \mathrm{C}\left(10^{\circ} \mathrm{C} / \mathrm{sec}\right)$ using the Hitachi Blaze heating holder in the SU9000. 
1) He ion irradiation of SC olivine particles. TEM imaging shows the presence of vesicles in the olivine grains after $0.5 \mathrm{dpa}$, and then a progressive increase of the number of vesicles with irradiation up to 1 dpa. The vesicles have sizes that range a few $\mathrm{nm}$ up to $\sim 50 \mathrm{~nm}$ in diameter. Secondary electron (SE) images show depressions, confirming that the vesicles are located on the surfaces of the grains. Our observations are similar to He irradiation of SC olivine at lower energy (12 keV) [9].

2) Xe ion irradiation of SC olivine and synthetic hibonite particles. TEM imaging of the hibonite particles after 0.5 and 1 dpa irradiation did not show any visual change to the surface morphologies or grain microstructure. However, after $2 \mathrm{dpa}$, SE images indicate the presence of vesicles on the surface of some grains. To determine whether the atomic structure was affected by irradiation, we acquired selected-area electron-diffraction (SAED) patterns from one grain in the same orientation before irradiation and after irradiation to $2 \mathrm{dpa}$. The SAED pattern prior to irradiation shows that the material contains long-range order, as revealed by discrete reflections, but minor streaking along $\mathrm{c}^{*}$ suggests some local stacking disorder. In comparison, the SAED pattern acquired after irradiation shows less intensity near to the forward-scattered beam but nonetheless still contains discrete reflections, indicating that it still has the long-range order. Thus, damage appears to be localized in the hibonite and irradiation has not significantly affected the bulk atomic structure.

Contrary to hibonite, the olivine grains do not show any modification of surface morphologies. However, comparison of the TEM imaging of olivine before and after irradiation suggests that the grains were at least partially amorphized after $0.5 \mathrm{dpa}$ of Xe irradiation.

Our observation of the (partial) amorphization of olivine is consistent with TEM studies of circumstellar silicate and oxide grains. Whereas most of the presolar oxides so far identified are crystalline, a large fraction of silicate grains is amorphous or nanocrystalline. The data presented herein suggest that the higher abundance of amorphous presolar silicates reflects their tolerance to radiation rather than their condensation as amorphous grains in their host circumstellar envelopes [10].

\section{References:}

[1] C Floss and P Haenecour, Geochemical Journal 50 (2016), p. 3.

[2] A Nguyen et al., The Astrophysical Journal 818 (2016), p. 51.

[3] Zega et al., The Astrophysical Journal 808 (2015).

[4] R Stroud et al., Lunar and Planetary Science Conference 45 (2014), abstract \#2806.

[5] C Vollmer et al., The Astrophysical Journal 769 (2013), p. 61.

[6] R Stroud et al., Science 305 (2004), p. 5689, p. 1455.

[7] H Ishii et al., Proceedings of the National Academy of Sciences 115 (2018), p. 26, p. 6608.

[8] R Christoffersen and LP Keller, Meteoritics \& Planetary Science 46 (2011), p. 7, p. 950.

[9] Thompson et al., Lunar and Planetary Science Conference 50 (2019), abstract \#1425.

[10] This work is supported by NASA Grant NNX15AD94G for the NExSS "Earths in Other Solar Systems" program, and NASA grant NNX15AJ22G. We are grateful to Hitachi High-Technologies for support with the SU9000 measurements. The use of the IVEM-Tandem facility was supported by the U.S. Dept. of Energy, Office of Nuclear Energy under DOE Idaho Operations Office Contract DEAC07-051D14517 as part of a Nuclear Science User Facilities experiment. We thank L. P. Keller for providing the synthetic hibonite sample. 